Revista de Comunicación y Salud, 2022 Vol. 12, 45-58

Editado por Cátedra de Comunicación y Salud

ISSN: 2173-1675

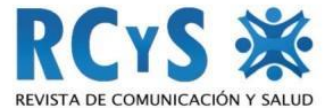

Enviado 01/06/2021

Aprobado 10/11/2021

Publicado 03/01/2022

\title{
Trascendencia de la comunicación en la calidad de vida del adulto mayor en el distanciamiento social por covid-19
}

\section{Transcendence of communication in the quality of life of the elderly adult in social distancing by COVID-19.}

Julio Hernández Falcón.

Universidad Nacional Autónoma de México. México.

Julio30falcon@comunidad.unam.mx

Araceli Jiménez Mendoza.

Universidad Nacional Autónoma de México. México. ajimenez55070@comunidad.unam.mx

Iñiga Pérez Cabrera.

Universidad Nacional Autónoma de México. México. inigapc@comunidad.unam.mx

\section{Cómo citar el artículo}

Hernández Falcón, J., Jiménez Mendoza, A. y Pérez Cabrera, I. (2022). Trascendencia de la comunicación en la calidad de vida del adulto mayor en el distanciamiento social por COVID-19. Revista de Comunicación y Salud, Revista de Comunicación y Salud, 12, 45-58. http://doi.org/10.35669/rcys.2022.12.e288

\section{Resumen}

Introducción. La persona adulta mayor ve afectadas sus funciones, auditivas, visuales y de movilidad; enfrenta limitaciones para incorporarse a los grupos sociales y la autodeterminación, que en ocasiones le posicionan en condición de dependencia para cubrir sus necesidades básicas afectando su calidad de vida. La comunicación es fundamental para el desarrollo de sus funciones, en el caso del distanciamiento social por COVID-19, la afectó la relación interpersonal y el intercambio de información. La comunicación evita la disminución de las funciones cognitivas y el deterioro paulatino que propicia la exclusión social. S1 Investigación de revisión evaluativa, documental, 
centrada en la comunicación, calidad de vida, adulto mayor. La revisión de la literatura publicada de 2019-2020, en el contexto de la pandemia por Covid-19, con base en los descriptores de "Calidad de vida", "Adulto mayor", "Comunicación", "aislamiento social", "COVID 19", "soledad". La estructura metodológica y conceptual, se basó en el análisis: 7 trabajos: 1 revisión sistemática, 3 estudios descriptivos, 2 ensayos y 1 tesis de grado. Resultados. Con base en los núcleos temáticos, se contrastaron planteamientos teóricos sustentados en el fomento de la comunicación y la diversificación a través de las TICs, calidad de vida, en el distanciamiento social por COVID-19, que constituye un riesgo para la salud y bienestar en el adulto mayor. Discusión. Los recursos tecnológicos para la comunicación son esenciales en la pandemia por COVID-19. Sin embargo, existe la brecha generacional, la carencia de recursos económicos para adquirirlos, entre otros factores que obstaculizan establecer una comunicación satisfactoria.

Palabras clave: Comunicación, Adulto mayor, calidad de vida, distanciamiento social, COVID-19.

\begin{abstract}
Introduction. In the larger adult persona their functions, auditory, visual and mobility are affected; it faces constraints in joining social groups and self-determination, which on occasions find themselves in a condition of dependence to cover their basic needs, affecting their quality of life. Communication is fundamental for the development of its functions, in the case of social distancing by COVID-19, the affect on the interpersonal relationship and the exchange of information. Communication prevents the decrease in cognitive functions and the gradual deterioration that leads to social exclusion. Method. Investigation of evaluative, documental, communication-centered review, quality of life, larger adult. The review of published literature from 2019-2020, in the context of the pandemic by Covid-19, based on the descriptors of "Calidad de vida", "Adulto mayor", "Comunicación", "social isolation", "COVID 19", "soledad". The methodological and conceptual structure, based on the analysis: 7 works: 1 systematic review, 3 descriptive studies, 2 essays and 1 grade thesis. Results. Based on the thematic nuclei, theoretical foundations were contrasted based on the promotion of communication and diversification through ICTs, quality of life, and social distance by COVID-19, which constitutes a risk for health and wellness in the major adult. Discussion: Technological resources for communication are essential in the pandemic by COVID-19. However, there is a generation gap, a lack of economic resources to acquire them, among other factors that hinder the establishment of satisfactory communication.
\end{abstract}

KEYWORD: Communication, Elderly, quality of life, social distancing.

Revista de Comunicación y Salud, 2022, Vol. 12, 45-58 
Trascendencia de la comunicación en la calidad de vida del adulto mayor en el distanciamiento social por COVID-19

\section{INTRODUCCIÓN}

La OMS (1996) durante el Foro Mundial de la Salud, acuñó la siguiente definición de calidad de vida: "La percepción que un individuo tiene de su lugar en la existencia,en el contexto de la cultura y del sistema de valores en los que vive y en relación con sus objetivos, expectativas, normas y preocupaciones»; sin duda es una definición compleja que incluye tanto elementos objetivos como subjetivos.

La calidad de vida asociada a la personalidad, la percepción del bienestar y el gradode satisfacción vivencial tiene un carácter predominantemente subjetivo resultante de la interacción humana con la atención social de las necesidades de vivienda, alimentación, vestido, educación y ejercicio de la libertad; así como de la autonomía. En el caso del adulto mayor significa el logro del reconocimiento a partir de las relaciones humanas significativas y contrarias al aislamiento social (Vera, 2017)El concepto de envejecimiento activo, según la OMS constituye "el proceso de optimización de las oportunidades de salud, participación y seguridad con el fin de mejorar la calidad de vida a medida que las personas envejecen" (Varela, 2016).

En el adulto mayor, tomar parte en forma activa y comprometida de sus acciones a través de la comunicación, constituye un elemento sustantivo de su calidad de viday facilita de manera importante la relación con la familia, amigos y entorno.

El adulto mayor experimenta cambios biológicos, emocionales y sociales, que se vinculan a los estilos de vida; la convivencia social y familiar que en conjunto mantienen o vulneran su autonomía (Loredo, Gallegos, Xeque, Palomé \& Juárez, 2016).

En el adulto mayor existen además dos elementos subjetivos de importancia: los cognitivos y los afectivo-volitivos. Los primeros relacionados con los significados que la persona atribuye a los hechos y los segundos referidos a los sentimientos, los estados de ánimo y los aspectos que le impulsan a la acción.

En este sentido, la comunicación definida como "la capacidad que tiene el hombre de simbolizar y responder de diferentes maneras ante un estímulo, que le lleva a preguntar, responder, compartir significados en una interacción de grupo o en un discurso que le sostiene y le anima la vida, además de ser el motor para la expresiónde la actividad social" (Ortiz, 2009).

La integración plena de la comunicación a los grupos sociales y su papel activo en las mismas es el tejido social y depende de las propias redes funcionales de la comunicación en las que se encuentra inmersa la persona. Contrario a lo anterior la masificación e indiferencia rompe con la relación humana que en muchas ocasiones crea los estereotipos de los propios AM (CEPSIGER, 2001).

De esta manera, la comunicación resulta tener un carácter vital ligado a las necesidades tanto materiales como inmateriales de las personas, su autonomíalibertad y autoestima, por lo que no solo tiene un alcance de intercambio de

Revista de Comunicación y Salud, 2022, Vol. 12, 45-58 
Trascendencia de la comunicación en la calidad de vida del adulto mayor en el distanciamiento social por COVID-19

mensajes o ideas; es decir además la comunicación dota de sentido y liga las historias de vida con las experiencias, actuaciones, intereses, emociones y vivencias culturales de cada persona y particularmente en el AM.

En el estudio de la comunicación de Arias, et al (2017), su valor se centra en el sentido de la comprensión del bienestar comunicativo, que contribuye a la calidad de vida de la persona mayor, descartando a la vez el estigma de "ser viejo", sustento de la discriminación a que es condenado el AM.

En el adulto mayor es necesaria la comunicación para comprender sus necesidades dado que la capacidad comunicativa se reduce (Benítez y Benítez, 2019), y en muchos casos se encuentra comprometida la función auditiva que implica disminución de la comunicación e interacción con su entorno y requiere paciencia ydisposición por parte de los interlocutores.

Además, una comunicación de calidad es necesaria en la atención apropiada del personal sanitario (Serra, 2003), al igual que el trato humano con empatía, información clara y comprensible con el tiempo suficiente a cada persona (Pons,2006).

En el envejecimiento la disminución orgánica incrementa las desventajas relacionadas con el funcionamiento neuropsicológico, lo que es posible gracias a la tecnología actual para suplir las diversas limitaciones y lograr que el envejecimiento se convierta en posibilidad de optimizar los propios recursos de comunicación. Más aun los actuales avances científicos han dado argumentos en favor de la plasticidad cerebral asociada a la reorganización y modelaje (neuroquímico y neuroanatomico) que establece y fortalece las conexiones sinápticas y que inducen una verdadera regeneración neuronal (Tirro,2016).

A los antecedentes antes descritos se agrega, la implementación de estrategias de distanciamiento social propuesta por la OMS, como la norma para de evitar la propagación del virus SARS-CoV-2 entre kpoblación a nivel mundial (De la Mora, 2020). En ese contexto, las mediddel distanciamiento social y el aislamiento en casa han reducido las actividades económicas y la movilidad de las personas.

Durante la pandemia por COVID 19, los adultos mayores se han visto restringidos en su interacción social lo que ha dado como resultado un confinamiento involuntario a partir de la prohibición de visitas familiares, que les priva del contacto personal y acentúa las desigualdades en el acceso a los servicios, incluidos los relativos a la salud.

Se reconoce que son los adultos mayores uno de los grupos más vulnerables a la enfermedad no solo por las características propias de la pandemia, sino además las condiciones propias de salud que incluyen la existencia de enfermedades crónicas, la presencia de síndromes geriátricos, la necesidad del contacto interpersonal estrecho para su cuidado, la situación de encierro junto a otras personas vulnerables y el diagnóstico muchas veces difícil por las respuestas atípicas propias de este grupo de edad (Cataldi, 2020).

Revista de Comunicación y Salud, 2022, Vol. 12, 45-58 
Trascendencia de la comunicación en la calidad de vida del adulto mayor en el distanciamiento social por COVID-19

En México afirman Moreno-Tamayo Sánchez-García, \& Doubova (2017) "se han experimentado diversos cambios poblacionales que han debilitado la estructura y la dinámica de las redes familiares y de amigos, por lo quelos AM son susceptibles a sufrir aislamiento social".

El mismo estudio resalta la prevalencia del aislamiento social más elevado para el caso de las mujeres (40.3\%) que para los hombres (35.5\%). Ellas mostraron menornivel educativo y menor propensión a vivir en pareja; mayor frecuencia de deteriorocognitivo, depresión, ansiedad y discapacidad en actividades básicas e instrumentales de la vida diaria (lbídem, 2017)

En México, Gonzales (2006) y colaboradores han hecho la evaluación del impacto de algunas variables asociadas a la calidad de vida de los ancianos mexicanos "que acuden regularmente a cuatro centros del Instituto Nacional de Personas Adultas Mayores (INAPAM) en la Ciudad de México. El estudio destaca, la estrategia orientada al problema o conducta (56\%); seguida de la valoración en un (36.6\%), y por último (7.4\%) la de las emociones; aspectos que implican comunicación y reconocimiento de las propias posibilidades vivenciales.

Por su parte León, Martos, Galiano (2020), a través de un estudio de revisión realizado en España afirma la necesidad de la comunicación con la persona AM, para reducir el aislamiento social y la soledad; siendo de valor la intervención en el proceso mismo de la comunicación para favorecer el envejecimiento sano.

En el caso de Herrera, Martínez y Navarrete (2013), al realizar un estudio de intervención comunitaria para mejorar la calidad de vida del AM encontraron que las dinámicas grupales, las técnicas del buen consejo, las charlas educativas incluidas a través de 12 sesiones de 2 horas, contribuyeron a mejorar la autoestima y la calidad de vida de los 20 participantes.

El objetivo de esta revisión es: Identificar la trascendencia de la comunicación en la calidad de vida del adulto mayor en el marco del distanciamiento social derivado de la pandemia por COVID 19.

\section{MÉTODO}

Estudio de revisión evaluativa que integra de manera pormenorizada y crítica información (Vera CO, 2009) acerca de la calidad de vida del adulto mayor, a partir de la bibliografía publicada (evidencia científica) en 2019-2020, en el marco de la pandemia de Covid-19.

Los participantes en su carácter experto aportan en el estudio la perspectiva propia y la resultante de la revisión de cada uno de los trabajos.

Con base en la metodología, se realizó la búsqueda, a partir de la definición de los descriptores de "Calidad de vida", "Adulto mayor", "Comunicación", "aislamiento social", "COVID 19", "soledad", los cuales fueron combinados, para la selección de documentos

Revista de Comunicación y Salud, 2022, Vol. 12, 45-58 
Trascendencia de la comunicación en la calidad de vida del adulto mayor en el distanciamiento social por COVID-19

electrónicos, los criterios para la selección de la literatura fueron título, estructura conceptual, metodológica y resultados. Se incluyen un total de 7 trabajos: 1 revisión sistemática, 3 estudios descriptivos, 2 ensayos y 1 tesis de grado.

En el procesamiento del fenómeno estudiado Tamayo (2006), se organizaron los artículos para el análisis por: título del artículo, autor, año, revista, información de la revista, problema de investigación, objetivos, tipo de investigación, método, descripción y tamaño de la muestra, instrumentos utilizados, resultados y núcleo temático.

\section{Resultados}

Se seleccionaron siete trabajos en español procedentes de: España, Perú, Cuba y México.

La revisión sistemática realizada de nueve artículos indexados, en PubMed, LATINDEX, Redalyc, Scielo, Scopus, Dialnet y Google Scholar por Vega R.J.A, Ruvalcava L.J.C, Hernández, Acuña, López (2020), en fuentes oficiales nacionales e internacionales, se enfocó en los principales ejes relacionados con la situación económica y laboral, la salud, así como los factores de riesgo generales para la población y específicos para las personas adultas mayores. Los autores concluyen que los adultos mayores conforman el grupo de mayor impacto negativo ante el SARSCov2 y COVID-19, su vulnerabilidad se sustenta por la edad, situación económica, laboral y enfermedades asociadas al grupo etario que pertenecen. La soledad incrementa el riesgo ante la necesidad de salir en busca de víveres, el no apoyarles potencializa el daño a la salud.

En el estudio transversal analítico de González, Norabuena, Olortegui. (2020), en el que la población estuvo conformada por 71 adultos mayores comprendidos entre 60 y 100 años. Se realizó una encuesta virtual a través de las redes sociales del 1 de al 31 de julio del 2020 utilizando el índice de Barthel. Encuestados virtualmente del 01 al 31 de julio del 2020, que aceptaron voluntariamente responder el cuestionario directamente, o a través de un familiar o cuidador, después de los 100 días de confinamiento obligatorio decretado por el Gobierno peruano.

Los resultados demuestran que el confinamiento social conlleva a una disminución de la actividad física impactando negativamente en la autonomía personal del adulto mayor. El $84.5 \%$ ha experimentado dolor musculo esquelético durante el confinamiento, el grado moderado de dolor en el $66.2 \%$, el grado severo en el $16.9 \%$ de ellos. El dolor está relacionado con factores como las comorbilidades, la falta de movimiento, la depresión, ansiedad y alteraciones en el sueño.

Callís, Guarton, Cruz, Armas (2021), en un estudio descriptivo, transversal, realizado entre abril y mayo de 2020, en el Policlínico "Josué País García", de Santiago de Cuba, en el que participaron 154 adultos mayores, mediante entrevista semiestructurada, concluyen que el aislamiento social, como medida para evitar el contagio, ha repercutido en la salud mental de los adultos mayores solos. Predominaron los niveles

Revista de Comunicación y Salud, 2022, Vol. 12, 45-58 
normales y leves de ansiedad y depresión, sin embargo también presentaron un nivel severo; y aunque no existió hegemonía deun grado extremo de estrés, la mayoría de los adultos mayores mostraron alteraciónen los niveles de estrés, lo cual pudiera darse por la concientización de su propia vulnerabilidad ante la pandemia, por la edad y la comorbilidad que presentaban; la incertidumbre de cuando retomar su vida y el conocimiento de las repercusiones sociales del COVID-19.

Naranjo, Mayor, Rivera , González (2021), realizaron un estudio descriptivo, transversal, entre mayo y junio de 2020 en 100 adultos mayores del policlínico Universitario "Dr. Rudesindo Antonio García del Rijo", Sancti Spíritus. Con base en una entrevista semiestructurada; la aplicación de la Escala de Ansiedad y Depresión Hospitalaria (HAD) de Carmen Terol Cantero $M$ et al y; la Escala de estrés percibido en línea de Campo Arias, et. al (2017), concluyen que la soledad como emoción y el aislamiento como condición estructural en la que viven muchos de los adultos mayores, juegan un papel importante frente a su capacidad de responder a las enfermedades contagiosas como la COVID-19.

El aislamiento social, como medida para evitar el contagio, ha repercutido de forma significativa en la salud mental de los adultos mayores solos, con respuestas emocionales como los trastornos leves de ansiedad y depresión, y marcada alteración en los niveles de estrés.

En el ensayo de Sánchez R, Sánchez F (2020), cuyo objetivo se enfocó en, identificar los problemas a los que se enfrenta el adulto mayor en el confinamiento y exponer algunas de las propuestas e intervenciones que se han llevado a cabo para proteger a este sector de la población, plantean que la aparición del Covid-19 ha producido un impacto significativo en los adultos mayores que se han visto afectados en mayor medida en comparación con el resto de la población. No sólo han sufrido una mayor mortalidad y han tenido mayores complicaciones en el desarrollo de la enfermedad, sino que han soportado las consecuencias más graves. La soledad y el aislamiento del adulto mayor en estos momentos han producido efectos devastadores. La paralización de todas sus actividades ha consecuencias físicas y psicológicas que están por evaluar. Para ello se propone establecer estrategias de atención integral al adulto mayor.

El ensayo crítico elaborado por Silva (2020), inicia el planteamiento a través de señalar que el principal riesgo sistémico de los adultos mayores en México, Brasil y EUA, durante la pandemia por COVID 19, han sido sus propios gobiernos, debido a que los adultos mayores, como el resto de la población, no tienen ningún tipo de injerencia sobre las decisiones epidemiológicas de sus gobiernos.

La pandemia por COVID 19 en México, se ha esparcido por toda la nación y en el caso de los adultos mayores, el reto es buscar proteger o controlar en lo posible el mayor número de riesgos sistémicos. 
Trascendencia de la comunicación en la calidad de vida del adulto mayor en el distanciamiento social por COVID-19

Estudio de caso clínico a una persona adulta mayor del género femenino desarrollado por García (2021), a través de la instrumentación de la Historia clínica, el test de ansiedad de Hamilton y el Inventario de depresión de Beck, concluye que la adulta mayor antes del aislamiento social por covid-19, tenía un ritmo de vida diferente, cuando fue diagnosticada con hipertensión y artritis su vida cambio, entro en una fase de negación, con la llegada del confinamiento la situación empeoro, debido a que surgieron ideas de posibles contagios, preocupación por su familia, se aisló, presento pérdida del apetito y dificultad para conciliar el sueño. Debido a la sintomatología se procedió a la valoración mediante la Escala de Ansiedad de Hamilton, se obtuvo un resultado de 19, lo cual indica que es Leve. En el Inventario de depresión de Beck, se valora como depresión leve.

El confinamiento por Covid-19 influye en el trastorno depresivo de una adulta mayor debido a que es una problemática reciente y desconocida, en la que se encuentra expuesta a muchos cambios dentro de su entorno, dejando en evidencia sus puntos más vulnerables.

\section{Discusión}

La comunicación es la capacidad del hombre de simbolizar, responder, y compartir significados en una interacción grupal, en el que las redes sociales contribuyen a la salud del adulto mayor mediante la comunicación se transmite información, necesidades, intereses, cultura, conocimientos. Un discurso sostiene y anima la vida, además de ser el motor y expresión de la actividad social sin la cual el adulto mayor no puede realizarse como ser humano.

La Asamblea General de la Organización de los Estados Americanos (15 de junio de 2015) resalta como derecho de las personas adultos mayores la dignidad, independencia, participación, cuidado y autorrealización. Incluyendo la comunicación (escucha) y el ambiente propicio para la conversación (Vallet H.E, 2019).

Desde el punto de vista del cuidado el adulto mayor y la enfermera requieren una especial atención a los procesos de comunicación como elemento sustantivo de la calidad de la relación interpersonal y como puente con la realidad vivencial (Aguirre RDA, Elers MY, Oria SM, \& Pascual C Y. 2020).

Las evidencias revisadas muestran que existe una preocupación permanente por la comunicación y el impacto del covid 19 en las interacciones comunicativas de los adultos mayores. Más aun la pandemia SARS-COV2 ha mostrando la exclusión social y digital del adulto mayor (Ortiz V.JF et al, 2021). Concluyendo que la brecha digital debe servir como estímulo para los servicios de salud para generar soporte a la brecha de generaciones.

La presencia de la pandemia y la comorbilidad más frecuente en los adultos mayores eleva el estrés psicosocial y por lo tanto afecta la calidad de vida de los mismos. Son el aislamiento social y la incertidumbre elementos que más vulneran la comunicación

Revista de Comunicación y Salud, 2022, Vol. 12, 45-58 
(Velazco RVM, Limones AML, Suarez AGG, Reyes VH y Delgado MVE, 2020).La comunicación es una necesidad de primer orden en el ser humano y por lo tanto en el adulto mayor para mantener las redes de socialización, reducir el aislamiento social y la soledad; por lo cual es de gran valor la intervención en el proceso comunicativo para favorecer el envejecimiento sano en todas las esferas de la persona.

En el estudio de (Vera, M., 2007), los adultos mayores le dan significado a su calidad de vida a partir de la satisfacción de sus necesidades de comunicación e información.

La integración plena a los grupos sociales y el papel activo en las mismas procedende la comunicación; así como el tejido social depende de las propias redes funcionales de la comunicación en las que se encuentra inmerso el viejo.

En la intervención de la calidad de vida de los adultos mayores las redes sociales ylas nuevas tecnologías de comunicación e información potencian el desarrollo comunicativo del adulto mayor y los socializa.

Los programas de intervención para mejorar la calidad de vida de los adultos mayores deben considerar de manera prioritaria la optimización de los procesos de comunicación y autoestima; así como el cambio de paradigma del uso de medios impresos a nuevas tecnologías, en el estudio de Condeza, 2016, los adultos mayores externaron que utilizaban el internet en un (16,5\%) para noticias y espectáculos o cultura $(23,3 \%)$.

Es de destacar que si bien la comunicación es esencial para difusión de información confiable y fidedigna durante la pandemia; la realidad es que los grupos y edades son más o menos susceptibles en su uso y apropiación. De manera que su acceso y uso repercuten en la apreciación que las personas generan sobre la vida; así como de sus imaginarios (Quintero DD, 2020). El adulto mayor de esta forma se encuentra en situación vulnerable por su comunicación efectiva y la calidad de la misma.

En la actualidad, la calidad de vida se reconoce en sus diversas dimensiones para la satisfacción de las necesidades de las personas adultas mayores, ante la pandemia por COVID-19, las redes sociales y las TICs son el medio esencial para establecer comunicación; sin embargo la brecha generacional limitó el acceso y uso, aislando a esta población, como lo planteo también (Botero de Mejía y Pico Merchan, 2007), lo cual invita a la realización de un diagnóstico participativo a fin de diseñar estrategias y programas de intervención centrados en la comunicación acordes a sus requerimientos que contribuyan a su bienestar, proyectos en los que participen instituciones gubernamentales y civiles escuchen a las personas adultas mayores y las hagan participes de las acciones para mejorar la comunicación, acorde a los recursos personales, familiares y comunitarios para mejorar su calidad de vida. 
Trascendencia de la comunicación en la calidad de vida del adulto mayor en el distanciamiento social por COVID-19

\section{Conclusiones}

A partir de la lectura crítica de los documentos seleccionados se concluye:

- La calidad de vida a pesar de tener un componente subjetivo está supeditadoa las condiciones materiales de vida y salud.

- La comunicación está íntimamente ligada a la calidad de vida del adulto mayor lo que favorece la plena realización y autonomía en el contexto histórico social.

- El ejercicio pleno de los derechos humanos del adulto mayor depende del grado de integridad de los procesos de comunicación en el marco de la red social.

- La emergencia suscitada por la pandemia COVID 19 ha acentuado el proceso de aislamiento del adulto mayor ya sometido a los cambios generacionales y a los estereotipos culturales.

- El aislamiento social producto de la pandemia compromete los mecanismosde afrontamiento sano del adulto mayor; así como el acceso a los servicios, incluidos los de salud.

- Existen diferencias significativas de base en la susceptibilidad de hombres y mujeres adultos mayores que reproducen las diferencias culturales ya presentes de manera ancestral.

- Los programas de intervención para mejorar la calidad de vida de los adultos mayores deben considerar de manera prioritaria la optimización de los procesos de comunicación y autoestima.

- La intervención en la calidad de vida de los adultos mayores debe aprovecharlas redes sociales y potenciar a través de las nuevas tecnologías de comunicación e información.

- La pérdida de capacidades funcionales también incluye disminución de la comunicación e interacción social.

Revista de Comunicación y Salud, 2022, Vol. 12, 45-58 
Trascendencia de la comunicación en la calidad de vida del adulto mayor en el distanciamiento social por COVID-19

\section{REFERENCIAS}

Aguirre RDA, Elers MY, Oria SM, \& Pascual C Y. (2020). Communication in nursing care with elderly people or their caregivers in a community of 10 de Octubre Municipality, 2018. Revista Habanera de Ciencias Médicas, 19(4). http://scielo.sld.cu/scielo.php?script=sci arttext\&pid=S1729-

519X2020000500015\&lng=es\&tlng=en

Botero de Mejía, Beatriz Eugenia; Pico Merchán, María Eugenia, (2007) Calidad de vida relacionada con la salud (cvrs) en adultos mayores de 60 años: una aproximación teórica. Universidad de Caldas Colombia. Revista Hacia la Promoción de la Salud. 12, 11-24

Cervantes N.P (2016) Calidad de vida y comunicación. UVM. https://bit.ly/3y0Npl8

Condeza, Ana Rayén, Bastías, Gabriel, Valdivia, Gonzalo, Cheix, Consuelo, Barrios, Ximena, Rojas, Rodrigo, Gálvez, Myrna, \& Fernández, Francisco. (2016). Elderly in Chile: describing their needs for preventive health communication. Cuadernos.info, (38), 85-04. https://dx.doi.org/10.7764/cdi.38.964

De la Mora D.G (2020) Aislamiento social y vigilancia para contener la pandemia de Covid 19: algunas acciones de gobierno y actores sociales. Notas de coyuntura del CRIM, 26. https://bit.ly/3rpMLel.

García S.A.A (2021). Confinamiento por covid-19 y su influencia en el trastorno depresivo de un adulto mayor. Universidad Técnica de Babahoyo. Disponible en: https://bit.ly/3x0KBD9

García-Vera, E. M., Robles-Rodríguez, A., Villegas-Bernabé, M. D. L., Figueroa- García, J., \& Etzel, A. (2016). La comunicación médico paciente ¿reto para el paciente geriátrico o para el médico familiar? Atención Familiar, 23(2), 63-66.

GEPSIGER (2001). Comunicación y envejecimiento. Ideas para una política. Colombia: Centro de psicología gerontológica. http://bitly.ws/dFRQ

González Celis, A L; Padilla, A (2006) Calidad de vida y estrategias de afrontamientoante problemas y enfermedades en ancianos de Ciudad de México Universitas Psychologica, 5 (3), 501-509. https://bit.ly/3wRsX4x

González ME, Norabuena M, Olortegui A. (2020) Autonomía personal del adulto mayor después de los 100 días de confinamiento por COVID-19. Perú:CASUS. 5(3), 138-144.

Herrera S.P.M, Martínez G.N y Navarrete R.C (2013). Intervención comunitaria para mejorar la calidad de vida del adulto mayor. Cuba: Revista Cubana de Medicina General Integral; 30(4),326-345 http://bitly.ws/dFRR

Revista de Comunicación y Salud, 2022, Vol. 12, 45-58 
Trascendencia de la comunicación en la calidad de vida del adulto mayor en el distanciamiento social por COVID-19

León L.M.I, Martos E.M, Galiano C.T. (2020) Técnicas de comunicación en las personas adultas mayores ante el aislamiento social y la soledad. España: Revista Española de Comunicación en Salud 2020, 11(2), 268-277. http://bitly.ws/dFRY

Loredo-Figueroa, M.T., Gallegos-Torres, R.M., Xeque-Morales, A.S., Palomé- Vega, G., \& Juárez-Lira, A.. (2016). Nivel de dependencia, autocuidado y calidad devida del adulto mayor. Enfermería universitaria, 13(3), 159-165. https://doi.org/10.1016/.reu.2016.05.002

Martínez, M. Á. M., \& Ibañez, L. M. (2012). La habilidad de comunicar: caminando hacia el paciente. Revista Española de Comunicación en Salud, 3(2), 158-166.

Moreno-Tamayo, K, Sánchez-García, S, \& Doubova, Svetlana V. (2017). Factores asociados con el aislamiento social en una muestra de adultos mayores con seguridad social. Salud Pública de México, 59(2), 119-120. https://doi.org/10.21149/8078

Naranjo H.Y, Mayor W.S, Rivera G.O, González B.R (2021).: Estados emocionales de adultos mayores en aislamiento social durante la COVID-19. Cuba Rev Información Científica. 100 (2). https://bit.ly/2V8fpV6

OMS (1996) La gente y la salud. ¿Qué calidad de vida. Ginebra: Foro Mundial de la Salud • Volumen 17. https://bit.ly/3eHwgoR

Ortiz M.J (2009) Comunicación interpersonal en el adulto mayor. Bogotá: Universidad Javeriana: Tesis de grado. http://bitly.ws/dFSd

Ortiz V.JF et al (2021) Aislamiento y efectos de la comunicación e inequidad en el adulto mayor. Universidad de Guanajuato. XXVI Verano de la ciencia. V.10 https://www.jovenesenlaciencia.ugto.mx/index.php/jovenesenlaciencia/article/vie $\underline{\mathrm{w} / 3285 / 2787}$

Pons X (2006) La comunicación entre el profesional de la salud y el paciente: aspectos conceptuales y guía de aplicación. Enfermería Integral. Colegio oficial de enfermería. Valencia N.73 https://bit.ly/3hVaBM3

Quintero DD (2020) Representaciones de la vejez, en tiempos de pandemia covid-19, en medios de comunicación de Colombia. Colombia: Pontificia Universidad Javeriana. (Tesis de Grado. Sociología). https://repository.javeriana.edu.co/bitstream/handle/10554/51969/Trabajo\%20de \%20grado\%20en\%20Sociologi\%cc\%81a\%20\%20Daniela\%20Quintero\%20D\%c3\%adaz.pdf? sequence=2\&isAllowed=y

Sánchez O.R, Sánchez V.J.F (2020) El aislamiento del adulto mayor por el COVID-19: consecuencias e intervenciones psicosociales durante la cuarentena. Salamanca: Studia Zamorensia, XIX file:///C:/Users/user/Downloads/Dialnet EIAislamientoDelAdultoMayorPorEICOVID19-7718175\%20(1).pdf

Revista de Comunicación y Salud, 2022, Vol. 12, 45-58 
Trascendencia de la comunicación en la calidad de vida del adulto mayor en el distanciamiento social por COVID-19

Serra Rexach, J. A. (2003). Comunicación entre el paciente anciano y el médico.In Anales de Medicina Interna 20 (2), 7-8. https://bit.ly/3xXaYLz

Silva C.M.D. (2020) Riesgo sistémico fundamental que el adulto mayor enfrenta anteel COVID 19 en México, Brasil y EUA. InterAm J Med Health. file:///C:/Users/user/Downloads/190-Article\%20Text-1032-21020210614\%20(1).pdf

Tirro V.I (2016) La vejez y el cerebro. Venezuela: Revista Nuevo Humanismo. 4(1), 7380. https://doi.org/10.15359/rnh.4-1.4

Vallet HE (2019) El Derecho de Comunicación entre abuelos y nietos. Algunas consideraciones desde el estudio de la violencia contra el adulto mayor. Transcontinental Human Trajectories. https://www.unilim.fr/trahs/index.php?id=1317\&lang=en

Varela P.L.F (2016) Salud y calidad de vida en el adulto mayor. Perú: Rev Peru MedExp Salud Publica 33 (2) https://doi.org/10.17843/rpmesp.2016.332.2196

Vega R.J.A, Ruvalcava L.J.C, Hernández P.I, Acuña G.M.R, López P.L (2020) La Salud de las Personas Adultas Mayores durante la Pandemia de Covid-19. Journal .5(7) 726-739. https://bit.ly/3iHeh31

Velazco RVM, Limones AML, Suarez AGG, Reyes VH y Delgado MVE (2020) Ansiedad en el adulto mayor durante la pandemia de COVID-19. Paraninfo digital año XIV N.32 http://www.ciberindex.com/index.php/pd/article/view/e32069d

Vera CO (2009) Como escribir artículos de revisión. Rev. Méd. La Paz 15 (1), 63-69 https://bit.ly/3xYWHhf

Vera M. (2007). Significado de la calidad de vida del adulto mayor para sí mismo y para su familia. Anales de la Facultad de Medicina, 68(3), 284-290. http://bitly.ws/dFSi

Vera, Martha. (2007). Significado de la calidad de vida del adulto mayor para sí mismo y para su familia. Anales de la Facultad de Medicina, 68(3), 284-290. http://www.scielo.org.pe/scielo.php?script=sci arttext\&pid=S1025$\underline{55832007000300012 \& \operatorname{lng}=\text { es\&tlng=es }}$

Revista de Comunicación y Salud, 2022, Vol. 12, 45-58 


\section{AUTORES}

\section{Julio Hernández Falcón}

Profesor de carrera asociado $\mathrm{C}$ de Tiempo Completo de la División del Sistema de Universidad Abierta y a Distancia de la ENEO-UNAM; Maestro en Investigación de Servicios de Salud; Miembro de la Línea de Investigación de Bienestar y cuidado, miembro de la Academia de Humanidades y Cuidado.

Orcid ID: https://orcid.org/0000-0002-0614-4342

\section{Araceli Jiménez Mendoza}

Profesor de carrera titular B, de Tiempo Completo de la División del Sistema de Universidad Abierta y a Distancia de la ENEO-UNAM; Miembro del Grupo de Cultura y Sociedad; Línea de Investigación Cuidado a la Vida y la salud de las personas; Sub línea Cuidado en las transiciones de vida. Integrante de la Academia de Investigación en la ENEO UNAM. Candidata Sistema Nacional de Investigadores CONACYT.

Orcid ID: https://orcid.org/0000-0001-5762-4314

\section{Iñiga Pérez Cabrera}

Profesor de carrera asociado $\mathrm{C}$ de Tiempo Completo de la División del Sistema de Universidad Abierta y a Distancia de la ENEO-UNAM, Decana de la Escuela, Dra. Miembro de la Línea de Investigación de Bienestar y cuidado, miembro de la Academia de Humanidades y Cuidado.

Orcid ID: http://orcid.org/0000-0002-5068-6325 